\title{
Avaliação comparativa do escoamento e tempo de presa do cimento MTA Fillapex ${ }^{\circledR}$
}

\section{Comparative assessment of flow and setting time of the MTA Fillapex $^{T M}$ sealer}

\author{
Gislaine Faraoni* \\ Meiryelen Silva Finger* \\ Michel do Carmo Masson ${ }^{* *}$ \\ Fausto Rodrigo Victorino ${ }^{* * *}$
}

\section{Resumo}

Na endodontia, o conhecimento das propriedades físicas dos cimentos endodônticos faz-se necessário para a realização de um adequado procedimento clínico. Objetivo: no presente estudo, o MTA Fillapex ${ }^{\circledR}$ foi comparado com o Sealer 26 $6^{\circledR}$ AH Plus ${ }^{\circledast}$ e Sealapex ${ }^{\circledast}$, em relação ao escoamento e ao tempo de presa. Materiais e método: para o teste de escoamento, em triplicata, cada cimento foi manipulado e depositado sobre uma placa de vidro e, logo após, foi colocada outra placa de vidro e um peso adicional de $120 \mathrm{~g}$ por 10 minutos. Com auxílio de um paquímetro digital, foram medidos os diâmetros maiores e menores dos cimentos, sendo obtida uma média aritmética. Para a obtenção do tempo de presa, em triplicata, cada cimento foi depositado em anéis de PVC. Após $30 \mathrm{~min}$, iniciaram-se as medidas com uma agulha tipo Gillmore de $100 \mathrm{~g}$ e 456,3 g, equivalentes ao tempo inicial e final respectivamente, sendo o seu resultado obtido após as marcas das aguIhas deixarem de ser visíveis nos cimentos. Resultados: o MTA Fillapex ${ }^{\circledR}$ apresentou uma taxa de escoamento maior do que a dos demais cimentos e chegou à presa inicial em um tempo maior, quando comparado com o $A H$ Plus $^{\circledR}$, e mais rapidamente que o Sealer $26^{\circledR}$. O cimento Sealapex ${ }^{\circledR}$ não tomou presa inicial e o MTA Fillapex ${ }^{\circledR}$ não tomou presa final, o que também foi evidenciado para o Sealapex $x^{\circledR}$. Conclusões: o cimento MTA Fillapex ${ }^{\circledR}$ está de acordo com a ADA quanto ao escoamento, e o tempo de presa inicial foi maior que o descrito pelo fabricante.

Palavras-chave: Obturação do canal radicular. Propriedades físicas. Endodontia.

\section{Introdução}

A escolha do cimento obturador é uma etapa crítica durante a fase de obturação dos canais radiculares, sendo o conhecimento de suas características de fundamental importância. Um cimento endodôntico ideal deve apresentar, entre outras propriedades, qualidades antissépticas permanentes, baixa viscosidade, bom escoamento, biocompatibilidade, baixa solubilidade, $\mathrm{pH}$ alcalino, estabilidade dimensional, tempo de trabalho adequado, radiopacidade, atividade bacteriostática e adesividade, além de não manchar as estruturas dentárias ${ }^{1}$.

A propriedade de escoamento de um cimento obturador consiste na sua capacidade de penetrar em irregularidades, ramificações do sistema de canais radiculares e nos túbulos dentinários. Quanto maior a fluidez do material, maior será sua capacidade de penetração; no entanto, também será maior a chance de ocorrer extravasamento do material e, consequentemente, proporcionar danos ao tecido periapical ${ }^{2}$.

O tempo de presa do cimento é outro parâmetro importante, pois, com base nele, é possível calcular o intervalo de tempo disponível para a obturação dos canais radiculares. Essa propriedade pode sofrer interferência de fatores como temperatura, relação entre pó e líquido, granulometria, meio ambiente e pH. De acordo com Alan et al. ${ }^{3}$, esse tempo não deve ser longo a ponto de prejudicar a conduta clínica,

\footnotetext{
Acadêmicas da Faculdade de Odontologia, Centro Universitário de Maringá (UNICESUMAR), Maringá, PR, Brasil. Participantes do Projeto de Iniciação Científica - PIC/UNICESUMAR.

Acadêmico da Faculdade de Odontologia, UNICESUMAR, Maringá, PR, Brasil.

*** Doutor em Endodontia, professor da Faculdade de Odontologia, área de Endodontia, UNICESUMAR, Maringá, PR, Brasil.
} 
pois assim pode haver a deterioração do cimento, favorecendo a penetração de agentes irritantes e a liberação de possíveis produtos tóxicos, e também não deve ser curto, para que o trabalho seja finalizado adequadamente.

Os cimentos obturadores são classificados, de acordo com os componentes da sua formulação, em: cimentos à base de resina; cimentos à base de óxido de zinco e eugenol, que contêm ou não medicamentos; cimentos que contêm hidróxido de cálcio e cimentos à base de ionômero de vidro.

$\mathrm{O}$ agregado de trióxido mineral (MTA) foi inicialmente introduzido na endodontia para o selamento de perfurações radiculares patológicas ou iatrogênicas e retro-obturações, por suas propriedades físicas, químicas e biológicas favoráveis ${ }^{4,5}$. Entretanto, o MTA não apresentava propriedades físicas compatíveis com o seu uso como cimento obturador no tratamento endodôntico. Com isso, uma nova formulação foi lançada no mercado, o MTA Fillapex ${ }^{\circledR}$ (Angelus, Londrina, PR, Brasil), um cimento obturador composto por MTA, resina de salicilato, resina natural, óxido de bismuto e sílica, que, de acordo com Camilleri ${ }^{6}$, possui melhores propriedades, como escoamento, tempo de presa e força de adesão.

Assim, o objetivo do presente estudo foi avaliar in vitro duas de suas propriedades, a capacidade de escoamento e o tempo de presa, com o objetivo de comparar os resultados com outros cimentos presentes há mais tempo no mercado: Sealer $26^{\circledR}$ (Dentsply, Petrópolis, RJ, Brasil), AH Plus ${ }^{\circledR}$ (Dentsply, DeTrey, Konstanz, Alemanha) e Sealapex ${ }^{\circledR}$ (Sybron Kerr, Washington, EUA).

\section{Materiais e método}

\section{Cimentos}

Os cimentos endodônticos avaliados foram divididos em: grupo I - MTA Fillapex ${ }^{\circledR}$ (Angelus, Londrina, PR, Brasil); grupo II - AH Plus ${ }^{\circledR}$ (Dentsply, DeTrey, Konstanz, Alemanha); grupo III - Sealer $26^{\circledR}$ (Dentsply, Petrópolis, RJ, Brasil) e grupo IV Sealapex $^{\circledR}$ (Sybron Kerr, Washington, EUA). Todos foram estocados e manipulados de acordo com as instruções dos respectivos fabricantes.

\section{Teste de escoamento}

A determinação do escoamento foi realizada com base na Especificação no 57 da American Dental Association $(\mathrm{ADA})^{7}$. Os cimentos foram manipulados na consistência ideal de acordo com as recomendações dos fabricantes, obtendo-se um volume de 0,5 $\mathrm{ml}$ ajustado em seringa Luer de 3,0 ml.

Cada cimento foi depositado no centro de uma placa de vidro com $10 \mathrm{~cm}$ de largura e $10 \mathrm{~cm}$ de comprimento. Após 180 segundos do início da manipulação, foi depositado sobre os cimentos um conjunto composto por uma placa de vidro e uma carga adicional de $120 \mathrm{~g}$. Após 10 minutos, o peso adicional foi removido e mediu-se os diâmetros maiores e menores dos discos por meio de um paquímetro digital ABSOLUTE (Mitutoyo Sul Americana, Suzano, SP, Brasil).

Duas condições foram necessárias para que o teste tivesse validade: a diferença entre os diâmetros maiores e menores dos cimentos não pôde ser superior a $1 \mathrm{~mm}$, e o disco teve que se apresentar uniformemente circular. O teste foi realizado em triplicata para cada grupo, obtendo-se uma média aritmética que representou o escoamento dos materiais.

\section{Tempo de presa}

Foram utilizados anéis de cloreto de polivinila (PVC) com $10 \mathrm{~mm}$ de diâmetro por $2 \mathrm{~mm}$ de altura para cada cimento testado, os quais permaneceram sobre uma placa de vidro de $1 \mathrm{~mm}$ de espessura por $25 \mathrm{~mm}$ de largura e $75 \mathrm{~mm}$ de comprimento. Os cimentos foram manipulados de acordo com as instruções dos fabricantes e colocados no interior dos anéis até que estes ficassem totalmente preenchidos. Todo o conjunto permaneceu em estufa a $37^{\circ} \mathrm{C}$.

Após 30 minutos do início da mistura, uma agulha tipo Gillmore de $100 \mathrm{~g}$ e ponta ativa de 2 $\mathrm{mm}$ de diâmetro foi colocada verticalmente sobre a superfície do material. O uso da agulha foi repetido até que não provocasse mais marcas no cimento testado, o que evidenciou a presa inicial do material. Posteriormente, uma agulha tipo Gillmore de $456,3 \mathrm{~g}$ e ponta ativa de $1,06 \mathrm{~mm}$ de diâmetro foi colocada verticalmente sobre a superfície do material. A inserção da agulha foi repetida até que parasse de marcar o cimento, o que indicou a presa final do material.

O tempo de endurecimento foi o decorrido entre o início da mistura e o momento no qual as marcas da agulha Gillmore deixaram de ser visíveis na superfície do cimento testado. O teste foi realizado em triplicata para cada grupo, obtendo-se uma média aritmética que representou tempo de presa dos materiais.

\section{Análise estatística}

Os dados encontrados para avaliação do tempo de presa não foram submetidos à análise estatística, pois alguns cimentos não tomaram presa. Os valores médios de escoamento foram submetidos à análise de variância (ANOVA) e ao teste de Tukey. $\mathrm{O}$ nível de significância adotado foi $\mathrm{p}<0,05$. 


\section{Resultados}

\section{Escoamento}

Os valores médios e respectivos desvios padrões para o escoamento dos cimentos encontram-se na Tabela 1.

Tabela 1 - Valores médios dos diâmetros em $\mathrm{mm}$ do escoamento dos cimentos obturadores

\begin{tabular}{|c|c|c|c|}
\hline Grupo & Média & Desvio padrão & Erro \\
\hline GI - MTA Fillapex ${ }^{\circledR}$ & $55,06^{a}$ & 0,645 & 0,37 \\
\hline Gll - AH Plus ${ }^{\circledR}$ & $47,63^{b}$ & 1,94 & 1,12 \\
\hline GIII - Sealer $26^{\circledast}$ & $43,58^{c}$ & 0,495 & 0,28 \\
\hline GIV - Sealapex ${ }^{\circledR}$ & $34,37^{d}$ & 1,031 & 0,59 \\
\hline
\end{tabular}

Letras diferentes indicam diferença significativa entre os grupos $(p<0,05)$. ANOVA e Post Hoc de Tukey.

O cimento MTA Fillapex ${ }^{\circledR}$ apresentou uma taxa de escoamento maior que AH Plus ${ }^{\circledR}$, Sealer $26^{\circledR}$ e Sealapex ${ }^{\circledast}$, respectivamente, e houve diferença significante estatisticamente entre todos os cimentos.

\section{Tempo de presa}

A Tabela 2 mostra os valores do tempo de presa para cada cimento avaliado.

\begin{tabular}{|c|c|c|}
\hline Grupo & Presa inicial & Presa final \\
\hline GI - MTA Fillapex ${ }^{\circledR}$ & $20 \mathrm{~h}$ e $11 \mathrm{~min}$ & NÃO \\
\hline Gll - AH Plus ${ }^{\circledast}$ & $19 \mathrm{~h}$ e $20 \mathrm{~min}$ & $23 \mathrm{~h}$ e $30 \mathrm{~min}$ \\
\hline GIII - Sealer $26^{\circledR}$ & $46 \mathrm{~h}$ e $54 \mathrm{~min}$ & $137 \mathrm{~h}$ e $50 \mathrm{~min}$ \\
\hline GIV - Sealapex ${ }^{\circledR}$ & NÃO & NÃO \\
\hline
\end{tabular}

Esses resultados evidenciaram que o MTA Fillapex $^{\circledR}$ chegou à presa inicial em um tempo maior quando comparado com o $\mathrm{AH} \mathrm{Plus}^{\circledR}$ e mais rápido que o Sealer $26^{\circledR}$. O cimento Sealapex ${ }^{\circledR}$ não chegou a tomar presa inicial, e O MTA Fillapex ${ }^{\circledR}$ não tomou presa final, o que também foi evidenciado no Sealapex ${ }^{\circledR}$.

\section{Discussão}

De acordo com Versiani et al. ${ }^{8}$, diferentes tipos de cimentos endodônticos vêm sendo usados em combinação com a guta-percha para preencher o canal radicular após o preparo biomecânico. Em relação a isso, Guttmann ${ }^{9}$ afirma que, independentemente da técnica escolhida, essa combinação é essencial para se obter um vedamento hermético do sistema de canais. Dessa forma, segundo Orstavik ${ }^{10}$, a escolha de um cimento obturador que ofereça boas proprieda- des físico-químicas torna-se essencial para que se tenha êxito no tratamento.

Os cimentos obturadores devem apresentar um escoamento que permita o selamento de irregularidades, canais acessórios e canais laterais. Por isso, a falta de escoamento prejudica a eficácia da obturação endodôntica ${ }^{11,12}$. No entanto, materiais com fluidez exagerada podem, durante a obturação endodôntica, extravasar para os tecidos periapicais, danificando-os. Dentre os fatores que influenciam no escoamento, a proporção pó-líquido, de fundamental importância, e o aumento da quantidade de líquido potencializam a ação irritante do cimento, tornando-o prejudicial no caso de extravasamento apical $^{13}$.

A ADA, em sua especificação $n^{0} 57$, propõe que o escoamento ideal para um cimento endodôntico seja igual ou maior que $25 \mathrm{~mm}$ de diâmetro. Para a avaliação do escoamento, Bernardes et al..$^{12}$ observaram uma média para o Sealer $26^{\circledR}$, o AH Plus ${ }^{\circledR}$ e o MTA Fillapex ${ }^{\circledR}$ de 29,51 mm, 37,47 mm e 27,65 $\mathrm{mm}$, respectivamente. Scelza et al. ${ }^{11}$ encontraram valores de $32,00 \mathrm{~mm}$ para o $\mathrm{AH}$ Plus $^{\circledR}$ e $39,00 \mathrm{~mm}$ para o Sealapex ${ }^{\circledast}$. Os resultados obtidos no presente estudo para o teste de escoamento foram de 55,06 mm para o MTA Fillapex ${ }^{\circledR}, 47,63 \mathrm{~mm}$ para o AH Plus $^{\circledR}, 43,58 \mathrm{~mm}$ para o Sealer $26^{\circledast}$ e $34,37 \mathrm{~mm}$ para o Sealapex ${ }^{\circledR}$, diferentemente dos outros estudos mencionados. Mesmo assim, porém, estão dentro do valor mínimo proposto pela especificação no 57 da $\mathrm{ADA}^{7}$. A alta fluidez do MTA Fillapex ${ }^{\circledR}$ pode comprometer seu desempenho clínico, pois aumenta a chance de seu extravasamento durante a obturação, o que pode acarretar dor intensa no pós-operatório, uma vez que, em recentes estudos biológicos, o MTA Fillapex ${ }^{\circledR}$ apresentou maior irritação em culturas de células $^{14}$ nas primeiras cinco semanas e moderada reação inflamatória nos primeiros sete dias em tecido conjuntivo ${ }^{15}$.

De acordo com Allan et al. ${ }^{3}$, o tempo de presa é uma propriedade importante, pois indica o intervalo de tempo disponível para a obturação após a manipulação do material. Idealmente, este não deve ser um tempo muito curto, para não dificultar a prática clínica, nem tão longo a ponto de favorecer a liberação de agentes irritantes. Segundo Orstavik et al. ${ }^{16}$ e Orstavik ${ }^{10}$, a temperatura ambiente, a umidade relativa e o tamanho das partículas têm grande influência sobre o tempo de presa: partículas menores aumentam a hidratação do material, acelerando o tempo de presa, e o contrário ocorre quando as partículas são maiores ${ }^{17}$.

Segundo os respectivos fabricantes, o tempo de presa para o Sealer $26^{\circledR}$ é de $48-60 \mathrm{~h}$ em temperatura ambiente; para o Sealapex ${ }^{\circledR}$, de aproximadamente $1 \mathrm{~h}$, com umidade relativa de $100 \%$; para o $\mathrm{AH}$ Plus $^{\circledR}$, de 8 h e para o MTA Fillapex ${ }^{\circledR}$, de 2 h no mínimo. No presente estudo, apenas o Sealer $26^{\circledR}$ esteve de acordo com o fabricante, tendo os outros cimen- 
tos apresentado tempo maior, ou, mesmo, não tomado presa, como foi o caso do Sealapex ${ }^{\circledR}$. Esses resultados comprovam que os cimentos MTA Fillapex ${ }^{\circledR}$ e Sealapex ${ }^{\circledR}$ necessitam de umidade para tomarem presa, uma vez que permaneceram em $37^{\circ} \mathrm{C}$ e $0 \%$ de umidade relativa. Quando em contato com água, partículas de silicato de cálcio do MTA formam um gel amorfo hidratado de silicato de cálcio, que polimeriza e endurece, formando uma rede contínua ${ }^{18}$. No estudo de Vitti et al. ${ }^{19}$, quando em presença de 95\% de umidade relativa do ar, o tempo de presa inicial do MTA Fillapex foi de 2 h e 27 min, significativamente menor que o encontrado no presente estudo. Deve-se ressaltar que o tempo de presa prolongado e a excessiva fluidez podem comprometer o resultado clínico no caso de extravasamento do cimento MTA Fillapex ${ }^{\circledR}$, pois seus componentes, como resina salicilato e sílica, relacionados com irritação celular $^{14}$, permanecerão em contato por maior tempo com os tecidos periapicais.

\section{Conclusão}

No presente estudo, o MTA Fillapex ${ }^{\circledR}$ apresentou maior taxa de escoamento que os demais cimentos, seguido do AH Plus ${ }^{\circledR}$, Sealer $26^{\circledR}$ e Sealapex ${ }^{\circledR}$.

$\mathrm{O}$ cimento MTA Fillapex ${ }^{\circledR}$ atendeu ao requisito mínimo sugerido pela ADA quanto ao seu escoamento, porém, seu tempo de presa inicial foi maior que o descrito pelo fabricante, na ausência de umidade.

\section{Agradecimentos}

Ao Centro Universitário de Maringá (UNICESUMAR), pela concessão do Projeto de Iniciação Científica (PIC), à Dentsply do Brasil e à Angelus Indústria de Produtos Odontológicos, por terem fornecido os cimentos endodônticos.

\section{Abstract}

In endodontics the knowledge of physical properties of endodontic cements is required to perform an adequate clinical procedure. Objective: in the present study, MTA Fillapex ${ }^{T M}$ was compared to Sealer $26^{T M}$, AH Plus ${ }^{T M}$ and Sealapex ${ }^{T M}$ regarding flow and setting time. Materials and method: for the flow test in triplicate, each cement was manipulated and placed on a glass plate, then another glass plate was placed with an additional weight of $120 \mathrm{~g}$, for 10 minutes. With the aid of a digital caliper the largest and smallest cement diameters were measured, and an arithmetic mean was obtained. To establish the setting time in triplicate, each cement was placed on PVC rings. After 30 minutes the measurements were started with a $100 \mathrm{~g}$ and $456.3 \mathrm{~g}$ Gillmore-type needle equivalent to the initial and final time respectively. The results were obtained after the brands of the needles were no longer visible in the cements. Results: the MTA Fillapex ${ }^{T M}$ presented a higher flow rate than other cements, and required more time to set up than AH Plus ${ }^{T M}$, but was faster than Sealer $26^{T M}$. The Sealapex ${ }^{T M}$ cement did not take the initial set, and the MTA Fillapex ${ }^{T M}$ did not take the final set, which was also evidenced for the Sealapex ${ }^{T M}$ cement. Conclusions: the MTA Fillapex ${ }^{T M}$ cement is in compliance with the $A D A$ in regards to flow, and the initial setting time was longer than that described by the manufacturer.

Keywords: Root canal filling. Physical properties. Endodontics.

\section{Referências}

1. Branstetter J, Fraunhofer JAV. The physical properties and sealing action of endodontic sealer cements: a review of the literature. J Endod 1982; 8(7):312-6.

2. Johnson WT, Guttmann JL. Obturation of cleaned and shaped root canal system. In: Cohen S, Hargreaves K. Pathways of the pulp. 9. ed. Philadelphia: Elsevier; 2007.

3. Allan NA, Walton RE, Shaffer M. Setting time for endodontic sealers under clinical usage and in vitro conditions. J Endod 2001; 27(6):421-3.

4. Gomes-Filho JE, Watanabe S, Bernabé PFE, Costa MTM. A Mineral trioxide aggregate sealer stimulated mineralization. J Endod 2009; 35(2):256-60.

5. Bogen G, Kuttler S. Mineral trioxide aggregate obturation: a review and case series. J Endod 2009; 35(6):777-90.

6. Camilleri J. Evaluation of selected properties of mineral trioxide aggregate sealer cement. J Endod 2009; 35(10):1412-7.

7. American National Standard. American Dental Association Specification $\mathrm{n}^{\circ} 57$ for endodontic sealing materials. Chicago: $\mathrm{ADA} ; 2000$.

8. Versiani MA, Carvalho-Junior JR, Padilha MI, Lacey S, Pascon EA, Sousa-Neto MD. A comparative study of physicochemical properties of AH Plus ${ }^{\mathrm{TM}}$ and Epiphany ${ }^{\mathrm{TM}}$ root canal sealants. Int Endod J 2006; 39(6):464-71.

9. Guttmann JL. Adaptation of thermoplasticized gutta-percha in the absence of the dentinal smear layer. Int Endod J 1993; 26(2):87-92.

10. Orstavik D. Materials used for root canal obturation: technical, biological and clinical testing. Endod Topics 2005; 12(1):25-38.

11. Scelza MFZ, Scelza P, Costa RF, Câmara A. Estudo comparativo das propriedades de escoamento, solubilização e desintegração de alguns cimentos endodônticos. Pesq Bras Odontoped Clin Integr 2006; 6(3):243-7.

12. Bernardes RA, Campelo AA, Silva Junior D, Pereira LO, Duarte MAH, Moraes IG, et al. Evaluation of the flow rate of 3 endodontic sealers: Sealer $26^{\mathrm{TM}}$, AH Plus ${ }^{\mathrm{TM}}$, and MTA Obtura $^{\mathrm{TM}}$. Oral Surg Oral Med Oral Pathol Oral Radiol Endod 2010; 109(1):e47-e49.

13. Holland D, Souza V, Juvenal M. Estudo histológico do comportamento de tecido subcutâneo do rato ao implante de alguns materiais obturadores de canal radicular. Influência da proporção pó-líquido. Rev Assoc Paul Cirur Dent 1971; 25(3):101-9.

14. Silva EJNL, Santos CC, Zaia AA. Long-term cytotoxic effects of contemporary root canal sealers. J Appl Oral Sci 2013; 21(1):43-7. 
15. Marques NCT, Neto NL, Fernandes AP, Rodini CO, Duarte MAH, Oliveira TM. Rat subcutaneous tissue response to MTA Fillapex $^{\circledR}$ and Portland Cement ${ }^{\circledR}$. Braz Dent J 2013; 24(1):10-4.

16. Orstavik D, Nordahl I, Tibballs JE. Dimensional change following setting of root canal sealer materials. Dent Materials 2001; 17(6):512-9.

17. Liu C, Shao H, Chen F, Zheng H. Effects of the granularity of raw materials on the hydration and hardening process of calcium phosphate cement. Biomaterials 2003; 24(23):410313 .

18. Abdullah D, Ford TR, Papaioannou S, Nicholson J, McDonald F. An evaluation of accelerated Portland cement as a restorative material. Biomaterials 2002; 23:4001-10.

19. Vitti RP, Prati C, Silva EJNL, Sinhoreti MAC, Zanchi CH, Souza e Silva MG, et al. Physical properties of MTA Fillapex Sealer $^{\mathrm{TM}}$. J Endod 2013; 39(7):915-8.

\section{Endereço para correspondência:}

Fausto Rodrigo Victorino

Rua Formosa, 489, Centro

86990-000 Marialva, PR

Fone: (44)3015-4534

E-mail: frvictorino@ig.com.br

Recebido: 12/03/2013. Aceito: 03/10/2013. 